OPEN ACCESS

Edited by:

E. Christien Michael Parsons,

University of Glasgow,

United Kingdom

Reviewed by:

Christopher Mull,

Dalhousie University, Canada

Andrew Kornblatt,

Online Ocean Symposium,

United States

*Correspondence:

André S. Afonso

afonso.andre@gmail.com

Specialty section:

This article was submitted to

Marine Conservation

and Sustainability,

a section of the journal

Frontiers in Marine Science

Received: 21 November 2019

Accepted: 21 July 2020

Published: 05 August 2020

Citation:

Afonso AS, Roque P, Fidelis $L$, Veras $L$, Conde A, Maranhão $P$,

Leandro S and Hazin FHV (2020)

Does Lack of Knowledge Lead

to Misperceptions? Disentangling

the Factors Modulating Public Knowledge About and Perceptions

Toward Sharks.

Front. Mar. Sci. 7:663.

doi: 10.3389/fmars.2020.00663

\section{Does Lack of Knowledge Lead to Misperceptions? Disentangling the Factors Modulating Public Knowledge About and Perceptions Toward Sharks}

\author{
André S. Afonso $0^{1,2 *}$, Pedro Roque ${ }^{1}$, Leonardo Fidelis ${ }^{1}$, Leonardo Veras ${ }^{3}$, Anxo Conde ${ }^{2}$, \\ Paulo Maranhão ${ }^{2}$, Sérgio Leandro ${ }^{2}$ and Fábio H. V. Hazin ${ }^{1}$ \\ ${ }^{1}$ Departamento de Pesca e Aquicultura, Universidade Federal Rural de Pernambuco, Recife, Brazil, ${ }^{2}$ MARE-Marine \\ and Environmental Sciences Centre, ESTM Polytechnic Institute of Leiria, Peniche, Portugal, ${ }^{3}$ Museu Dos Tubarões, \\ Fernando de Noronha, Brazil
}

Identifying sources of variability in public perceptions and attitudes toward sharks can assist managers and conservationists with developing effective strategies to raise awareness and support for the conservation of threatened shark species. This study examined the effect of several demographic, economic and socioenvironmental factors on the quality of knowledge about and perceptions toward sharks in two contrasting scenarios from northeastern Brazil: a shark hazardous coastal region and a marine protected insular area. Ordered logistic regression models were built using Likert data collected with a self-administered questionnaire survey $(N=1094)$. A clear relationship between education, knowledge and perceptions was found, with low education level and knowledge of sharks resulting in more negative perceptions toward these species. Prejudice toward sharks stemmed as a potentially limiting factor because the positive effects of other variables such as affinity for nature and specific knowledge about sharks were superseded by the effects of negative prejudice. Other practical factors such as age, economic level, and gender, also influenced respondent's knowledge and perceptions and could provide a guidance for optimizing socioenvironmental gains relative to public engagement efforts. Results also suggested that populations inhabiting regions with high shark bite incidence likely require distinct outreach methods because some factors underlying knowledge and perception dynamics exhibited structural differences in their effects when compared to the trends observed in a marine protected area. Altogether, public perceptions and attitudes toward sharks could be feasibly enhanced with educational development and nature experiencing strategies. Moreover, disseminating shark-specific knowledge across the society might catalyze support for the conservation of these species in a cost-effective way. This study provides a 
potentially useful socioenvironmental framework to deal with the human dimensions of shark management and to strengthen conservation policies aimed at promoting societal compliance with pro-environmental values, which is crucial to endow shark populations with effective protection from anthropogenic threats.

Keywords: awareness, conservation, Fernando de Noronha, Likert questionnaire, marine protected area, OLR models, Recife, shark hazard

\section{INTRODUCTION}

As aquatic predators, sharks play a decisive role in regulating trophic webs and maintaining ecosystem balance and structure (Stevens et al., 2000; Heupel et al., 2014; Hussey et al., 2015), in controlling the activity of their prey (Wirsing et al., 2007; Bond et al., 2019), and in promoting ecosystem connectivity (McCauley et al., 2012). Sharks provide ecosystem services of incommensurable value (Tavares et al., 2019) and their removal from the environment may prompt unpredictable and potentially dramatic processes along food webs (Myers et al., 2007; Casini et al., 2009; Bornatowski et al., 2014). However, an intensification of fishing pressure on shark species (Dent and Clarke, 2015) has led to substantial declines in their abundance (Dulvy et al., 2008; Davidson et al., 2015; Roff et al., 2018), with a global estimate of $\sim 100$ million sharks being caught by fisheries each year (Worm et al., 2013) and 25\% of all chondrichthyan species being currently threatened with extinction (Dulvy et al., 2014). This scenario is most worrisome because sharks are typically associated with low intrinsic rates of population increase (Cortés, 2000) and have little resilience to overexploitation. As such, recognition of the need to prioritize shark management and conservation has been spreading worldwide, prompting some countries and institutions to implement conservation measures (e.g., ICCAT, 2016; WCPFC, 2018; CITES, 2019).

A thorough understanding of shark bioecology and vulnerabilities is essential to derive adequate resource management measures toward sustainability (Yokoi et al., 2017), and researchers worldwide have been prolific in addressing knowledge requirements with direct applicability to shark conservation (e.g., Huveneers et al., 2015; Cardeñosa, 2019; Queiroz et al., 2019). However, all these ongoing efforts to ensure the sustainability of shark populations may not suffice (Simpfendorfer et al., 2011). Ultimately, the effective conservation of marine wildlife could depend on whether human societies are suitably supportive of, and compliant with, conservation goals (Bennett and Dearden, 2014). Societal mismatched values and attitudes may undermine conservation frameworks as they play against prospective management strategies developed during decision-making processes. For example, the efficacy of conservation policies aimed at terrestrial large-sized carnivores has been impaired by detrimental attitudes from local communities which perceived such species as potentially perilous (Treves and Karanth, 2003; Casey et al., 2005). In the marine realm, adverse public perceptions toward fatal shark bites have compelled authorities to implement shark culling programs targeting globally endangered and protected species (McCagh et al., 2015), which increases the amount of disturbance inflicted to their populations. Even regarding non-hazardous scenarios, sharks can be considered a nuisance and become prone to extirpation regardless of their conservation status, e.g., when they produce considerable economic loss trough depredation on fisheries catch (Mitchell et al., 2018).

Public perceptions could thus play a crucial role in shaping societal behaviors and attitudes toward wildlife and enabling support for conservation management. Examples of facilitated shark conservation have been reported after people perceptions and attitudes shifted toward more positive opinions about the socioeconomic value of live, free-ranging sharks and about the perceived value of recreational shark diving in opposition to fishing and other detrimental practices (Simpfendorfer et al., 2011; Whatmough et al., 2011). In turn, public perceptions and awareness toward shark conservation could be partially modulated by how much people are acquainted with these species. Previous research revealed that people with greater levels of knowledge about marine predators such as sharks or dolphins were more likely to adopt ecologically responsible behaviors and advocate for their conservation (Thompson and Mintzes, 2002; Barney et al., 2005; O’Bryhim and Parsons, 2015; Tsoi et al., 2016). Unfavorable public perceptions and insufficient knowledge about sharks might thus present a barrier to the development of shark conservation policies and contribute to the collapse of their populations. Understanding the mechanism that regulates the qualitative distribution of perceptions toward and knowledge about sharks across human societies is becoming an increasingly important prerequisite for successful marine conservation action.

The dynamics in public perceptions and knowledge about environmental subjects are typically very complex due to a high degree of heterogeneity within societies (Schultz and Zelezny, 2003) and to considerable geopolitical and cultural variability in human perceptions and attitudes toward nature and environmental sustainability across the globe (Leiserowitz et al., 2005). Knowledge, perceptions and, ultimately, proenvironmental behavior, seem to be influenced by a whole suite of factors of both extrinsic (e.g., demographics, socioeconomics, educational, and cultural context) and intrinsic (e.g., individual values, predispositions, preconceptions, motivations, and emotions) nature (Kollmuss and Agyeman, 2002; Steel et al., 2005; Acuña-Marrero et al., 2018). Prior experience with wildlife, such as sharks, and personal connection to environmental subjects are also important drivers of conservation awareness and pro-environmental behavior (Bögeholz, 2006; Friedrich et al., 2014; Skubel et al., 2019), however, worse perceptions and attitudes may arise when people are either faced with hazardous scenarios following shark bites incidents 
(Pepin-Neff and Wynter, 2018a) or exposed to negatively biased media's coverage of sharks (Muter et al., 2012). Understanding the drivers of knowledge and perceptions about sharks across regions with distinct relational perspectives toward these species could help to identify cognitive and emotional processes underpinning public awareness about and attitudes toward sharks, which would be useful to develop tailored, humanoriented strategies for shark conservation. On that account, this study aimed at scrutinizing the different types of human traits modulating the variability in public knowledge and perceptions about sharks, and at ascertaining potential shifts to be expected when comparing more favorable and more adverse scenarios concerning shark conservation. We sought to clarify some of the relational intricacies between sharks and humans by addressing opposite socioenvironmental scenarios built around either positive or negative interactions with sharks within the same analytical framework.

\section{MATERIALS AND METHODS}

\section{Study Site}

A survey to assess public knowledge about and perceptions toward sharks was conducted between June 2015 and December 2016 in Recife (REC) and in the Archipelago of Fernando de Noronha (FEN), Northeast Brazil. The two regions are peculiar in that they exhibit contrasting attributes regarding the relationship between sharks and humans. Located in the mainland, REC is the Pernambuco's state capital and has been associated with a serious spate of shark bites on humans since 1992 (Hazin et al., 2008), with a total of 65 incidents and 25 fatalities being recorded in the last 27 years (i.e., $\sim 2.4$ incidents per year on average). As a result, its population has endured significant socioeconomic losses including welfare deterioration and lifestyle inhibitions derived from local restrictions to ocean usage. Several shark species occur in this region (Afonso et al., 2014) but the tiger, Galeocerdo cuvier, and bull, Carcharhinus leucas, sharks are the species of major concern (Hazin and Afonso, 2014; Afonso et al., 2017a). On the other hand, FEN is a prestigious marine protected area (MPA) located $541 \mathrm{~km}$ off Recife where a thriving ecotourism industry benefits from a great abundance and diversity of charismatic megafauna, including sharks. FEN encompasses breeding and nursery habitats for some shark species (Garla et al., 2009; Afonso et al., 2016), rendering these animals easily observable by beach and ocean users. Despite a considerable overlap between shark and human populations and the occurrence of potentially dangerous species such as the tiger shark (Afonso et al., 2017b), shark hazard in FEN is quite low and no incidents have been reported except for some minor episodes in the last few years. We thus expected our sampling design to capture the two extremes of a gradient modulated by people's predispositions and reactions toward sharks.

\section{Sampling Procedure}

This study has been approved by the Committee for Ethics in Research with Human Beings of the Federal University of Pernambuco (permit \#50417915.6.0000.5208). Potential participants were randomly approached at public sites in REC and FEN to assess their willingness to take part in an anonymous survey after being informed that no personal data would be collected and that they would be able to quit their participation at any time. First, respondent eligibility was determined and people $<18$ years old or whose professions related directly with the environment were not included in the survey. Eligible individuals were then invited to complete a self-administered questionnaire comprised of 49 questions and assertions of different types. These included classic questions about demographic and socioeconomic variables $(N=5)$ and Likert-type assertions on knowledge about $(N=12)$ and perceptions toward $(N=7)$ sharks. A 5-level Likert scale was used, where 1 was coded as total disagreement, 3 was coded as neutral and 5 was coded as total agreement. Additional questions were introduced in the questionnaire for classifying respondents according to some relevant socioenvironmental and economic aspects. These included one free-text question about the emotions elicited by sharks and 19 multiple-choice questions, either ordered or categorical, which were used to derive proxies for respondent's affinity for nature $(N=6)$, feelings/prejudice toward sharks $(N=10)$ and more specific knowledge about sharks $(N=3)$. The proxy for more specific knowledge about sharks (hereafter referred to as specific knowledge) was deemed necessary for discriminating respondents exhibiting unusual acquaintance with shark-related issues and it differentiated from the Likert-derived knowledge about sharks in that it focused on more objective questions addressing quantitative metrics about shark hazard, biology and fisheries, which the general public would be unlikely to know. Further, 5 multiple-choice questions posed exclusively to FEN tourists were used to derive a proxy for economic level. The different questions were alphanumerically coded for cross-referencing purposes and are provided in Supplementary Table 1.

Three sampling treatments were defined according to the source of the data, i.e., data collected in Recife (hereafter referred to as treatment REC), and data collected among FEN residents and FEN tourists (hereafter referred to as treatments FENres and FENtour, respectively). The sampling effort was dimensioned in such a way that the number of samples accomplished in treatments REC and FENtour would, respectively, amount to about two and fourfold the number of samples accomplished in treatment FENres. Such a distribution in sampling effort derived from our expectation that heterogeneity within treatments would be greatest among FEN tourists and smallest among FEN residents.

\section{Statistical Analyses}

Initially, because treatment REC could also encompass people not based in Recife, we tracked respondents' area of residence and preliminarily assessed its effect on the demographic and socioenvironmental variability ascribed to treatment REC. For that, we used Pearson's $\chi^{2}$ tests and complementary Fisher's exact tests with simulated $p$-value based on 2,000 replicates to compare the distribution of several descriptors (i.e., age, education level, affinity for nature, prejudice/feelings toward sharks and specific 
knowledge about sharks) between respondents residing in Recife and respondents residing elsewhere.

A total of 10 potential predictors of knowledge about and perceptions toward sharks were considered. Besides sampling treatment, demographic and socioeconomic predictors included age, sex, education level (i.e., "Elementary," "High," and "Superior"), economic level (i.e., "Low," "Medium," and "High"), ocean proximity (i.e., whether respondents reside in littoral or interior states), and Brazilian geopolitical region of residence (i.e., Northeast, Central-West, Southeast, and South). There might be other religion or cultural factors that may potentially influence knowledge and perceptions about sharks. However, these were not investigated in the current study because the study focused on a regional population which, in general, shared the same sociocultural identity, besides that the ethics of ordering religions and ethnical aspects on a qualitative scale could be challenged by national institutions. The effects of such factors on knowledge and perceptions about sharks might nonetheless warrant further research, preferentially on a global scale in order to capture different shark-related ethnobiological and cultural scenarios.

Following Afonso et al. (2019), age data were binned into 3 age classes, i.e., <30, 30-44, and $>44$ years old for categorical analysis, but age was also interpreted as a continuous variable. Regarding socioenvironmental predictors, responses to multiple choice, non-Likert questions were coded following an ordinal scale, normalized by the number of available choices in each question, and averaged to derive an overall score for each respondent concerning his/her affinity for nature ("High," "Medium," and "Low"), feelings/prejudice about sharks ("Positive," "Neutral," and "Negative"), and specific knowledge about sharks ("High," "Medium," and "Low"). Free-text data to assess the emotions elicited by sharks in question J1 (Supplementary Table 1) were coded following a symmetric, 3-level ordinal scale, averaged for each respondent, and incorporated into the proxy for feelings/prejudice about sharks. Moreover, Likert assertions were enounced so that the margin levels ( 1 or 5 ) would match the correct or most positive response, but the assertions for which the correct/most positive response was " 1 " had their responses reversed prior to analysis in order to preserve " 5 " as the most favorable response.

To identify possible relationships among demographic, socioeconomic and socioenvironmental descriptors, Pearson's $\chi^{2}$ tests and complementary Fisher's exact tests with simulated $p$-value based on 2,000 replicates were performed pairwise to all possible combinations of descriptors. Subsequently, two ordered logistic regression (OLR) models were built to determine which variables would better explain variability in knowledge and perceptions. OLR models incorporated Likert knowledge and perception data as response variables and all the aforementioned descriptors as candidate predictor variables, with knowledge and perception responses being modeled separately. The Likert data were weighted as in Afonso et al. (2019) in order to penalize more complex assertions for which respondents would be more likely to reply randomly (Supplementary Table 1). A stepwise forward selection procedure based on the Akaike Information Criterion (AIC) was used for model building. Predictors for which Pearson's $\chi^{2}$ and Fischer's tests revealed significant relationships were not included simultaneously in the same model to avoid multicollinearity issues. Additionally, because the amount of interrelated predictors precluded the development of large models containing more than three variables, singlepredictor OLR models featuring each of the candidate predictor variables were developed as a complementary strategy to thoroughly explore the effect of every potential predictor on both knowledge and perception responses. All single-predictor OLR models incorporated first-order interactions between the respective predictor and all the remaining predictors except for economic level, region of residence and ocean proximity. With this strategy, we aimed at identifying inconspicuous trends which might have been superimposed by data structure.

Finally, the relationship between the two response variables (i.e., knowledge about and perceptions toward sharks) was examined by averaging both knowledge and perception Likert scores for each respondent and conducting Pearson's product moment correlations to the whole data set and to each sampling treatment separately. A linear regression featuring averaged Likert knowledge and perceptions as predictor and response variables, respectively, was also developed. Statistical analyses were conducted with R version 3.5.0 (R Development Core Team, 2018) using packages MASS (Venables and Ripley, 2002), and effects (Fox and Weisberg, 2019). Statistical significance was set at $p<0.05$.

\section{RESULTS}

\section{Data Description}

An overall total of 1094 respondents were included in the analysis, with 346 (32\%) representing treatment REC, 133 (12\%) representing treatment FENres, and 615 (56\%) representing treatment FENtour. Assuming a resident population size of $1.6 \times 10^{6}$ people in Recife and $3 \times 10^{3}$ people in FEN, as estimated by the Brazilian Institute for Geography and Statistics ${ }^{1}$ (Accessed on 19 September 2019), and an annual influx of $1 \times 10^{5}$ tourists in FEN, as reported by the local administration ${ }^{2}$ (Accessed on 19 September 2019), these sample sizes translate into margins of error of 5, 8, and $4 \%$ for treatments REC, FENres and FENtour, respectively. The whole sample was comprised of 577 female (53\%) and 517 male (47\%) respondents aged between 18 and 77 years old, with 3\% having completed the elementary school, $20 \%$ having completed the high school, $74 \%$ having obtained an academic degree and 3\% not having informed their education level. Altogether, respondents exhibited a seemingly good knowledge about sharks, with Likert level 5 being selected, on average, in $50 \%(S D=27 \%)$ of the replies and the remaining Likert levels oscillating between 9 and 15\%. Similarly, perceptions toward sharks were generally positive, with an average of $51 \%$ $(S D=17 \%)$ replies ascribed to Likert level 5 and the remaining levels ranging from 10 to $15 \%$. The frequency distributions of replies to each Likert question across the several factors analyzed are reported in Supplementary Figures 1-10.

\footnotetext{
${ }^{1}$ https://www.ibge.gov.br

${ }^{2}$ http://www.noronha.pe.gov.br
} 
Treatment REC included 38 (12\%) respondents who did not reside in Recife, but these were unlikely to affect the overall output of this treatment since no significant differences were detected between them and people residing in Recife for all variables tested but education level (Supplementary Table 2), which even so exhibited analogous distribution trends. Therefore, the whole REC data was used. Further, treatment FENtour included only $44(\sim 7 \%)$ of people coming from Pernambuco's mainland, whereas most tourists came from other states. The three sampling treatments exhibited some variability in demographic features. The age of respondents varied significantly across treatments (Table 1 and Supplementary Figure 11), with the mode of the age distribution increasing from 20 to 25 years in REC through $25-30$ and 30-35 years in FEN residents and tourists, respectively (Supplementary Figure 12). The sex ratio (male:female) also varied significantly across treatments

TABLE 1 | Pearson's $\chi^{2}$ test and Fisher exact test results for pairwise combinations of factors to assess their interdependency.

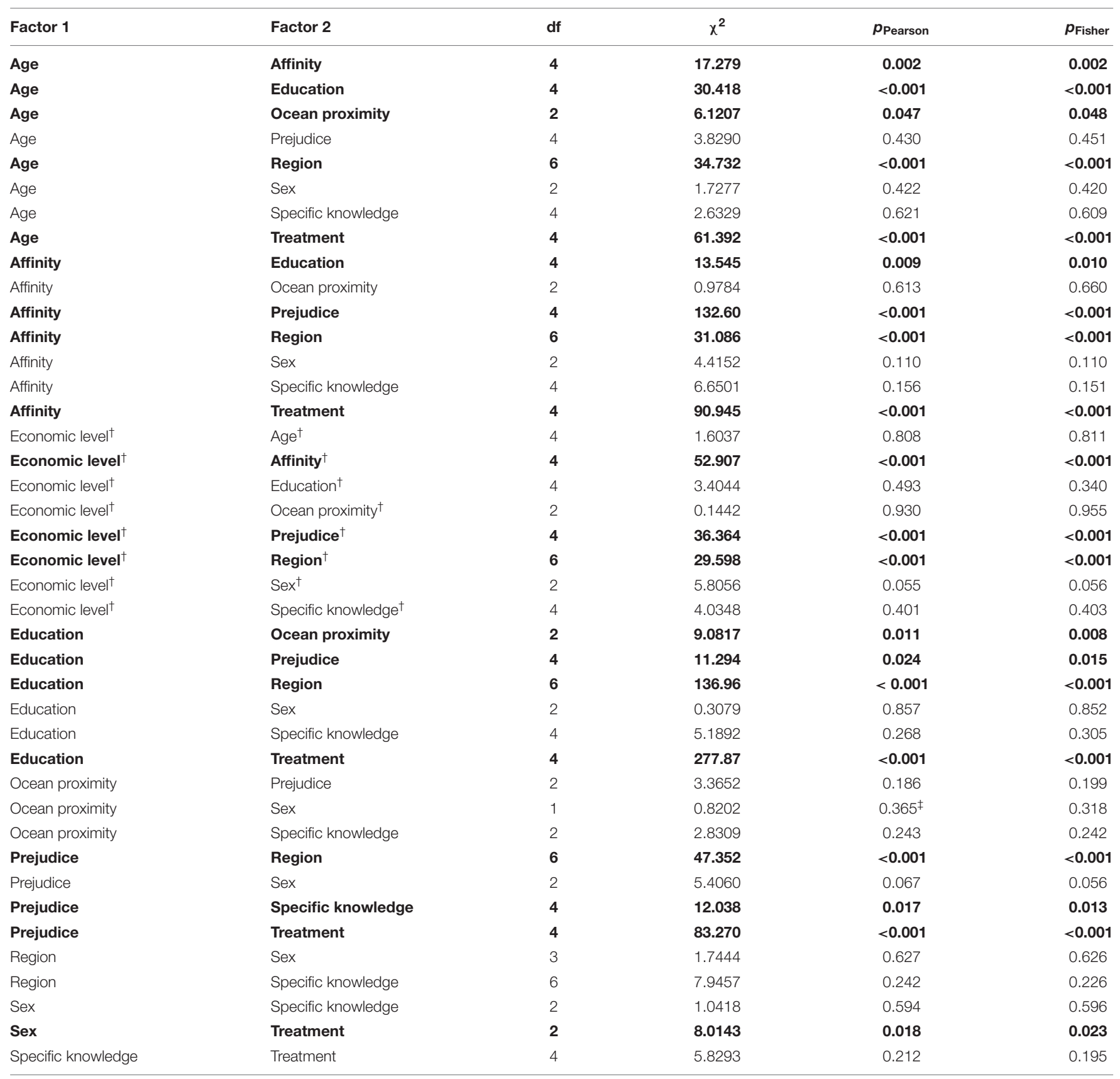

The number of degrees of freedom (df), the Persons's test statistics $\left(x^{2}\right)$, and $p$-values for Pearson's and Fisher tests ( $p_{\text {Pearson }}$ and $\left.p_{\text {Fisher }}\right)$ are reported. Statistically significant $(p<0.05)$ relationships between factors are highlighted in bold. Spurious relationships such as Treatment against Ocean proximity or Region and Ocean proximity against Region were not included. ${ }^{\dagger}$ Only FEN tourists were included in tests involving economic level. ${ }^{\ddagger}$ Pearson's test conducted with Yate's continuity correction. 
(Table 1 and Supplementary Figure 11), being 0.75:1 among FEN residents, 0.81:1 among FEN tourists, and 1.15:1 in Recife. Regarding education level, a significantly higher proportion of FEN tourists holding superior education contrasted with FEN residents, who exhibited the lowest education level of all treatments (Table 1 and Supplementary Figure 11). An unexpectedly high proportion (68\%) of respondents with superior education in REC indicates that the sample does not reflect the whole population from Recife, which could be due to sampling effort being concentrated at a beach frontline where many upper-class people reside. Notwithstanding, and presuming that a superior education would positively influence knowledge and perception levels, such a bias should at the most lead to an overestimation of these traits in treatment REC.

On the other hand, significant relationships between age distribution, education level, ocean proximity and region of residence were identified (Table 1 ). People $<30$ years old exhibited lower education level and were more represented in coastal states as well as in the Northeast region, where lower education levels were found (Supplementary Figure 11). Furthermore, the economic level of FEN tourists related significantly with region of residence (Table 1), with a lower economic profile being found in people coming from the Northeast (Supplementary Figure 13). Concerning socioenvironmental proxies, prejudice toward sharks was significantly more negative in REC compared with both FEN treatments and it was directly proportional to affinity for nature, specific knowledge about sharks (Table 1 and Supplementary Figure 11) and economic level (Supplementary Figure 13). In turn, affinity for nature was lowest in REC and highest in treatment FENres, besides demonstrating some regional variability evidenced by higher affinity scores ascribed to respondents from the Southeast (Table 1 and Supplementary Figure 11). Also, affinity tended to increase with age and economic level while decreasing with education level, being lowest among people with high-school graduation (Table 1 and Supplementary Figures 11, 13).

\section{Statistical Modeling Knowledge About Sharks}

Significant differences in knowledge about sharks across sampling treatments were revealed by single-predictor OLR modeling (Table 2), with the lowest and highest levels of knowledge being ascribed to treatments REC and FENtour, respectively (Figure 1). However, the model selection procedure indicated knowledge to be best predicted by the interaction of sampling treatment with specific knowledge about sharks added to ocean proximity. With this arrangement, knowledge in both FEN treatments tended to rise as specific knowledge about sharks increased, yet it did not change in REC treatment (Table 3 and Figure 2). Further, knowledge tended to be slightly greater in coastal states compared to interior ones (Table 3 and Supplementary Figure 14). The sampling treatments also differed in that FEN residents aged 30-44 years revealed better knowledge than younger and older residents, whilst respondents
$>44$ years old exhibited the best knowledge in the other two treatments (Supplementary Figure 15).

A thorough examination of variability in knowledge about sharks revealed additional effects by demographic, economic and socioenvironmental predictors. For instance, knowledge improved with increasing education level, with people holding a superior education degree being 56\% more likely to provide the correct answer than elementary-educated people (Table 2 and Figure 1). Also, the magnitude of this effect tended to be higher among people with little specific knowledge about sharks (Supplementary Figure 16) and became imperceptible among people with low affinity for nature (Supplementary Figure 17). On the other hand, a significant $(p<0.001)$ although mild improvement in knowledge with increasing age was evidenced, with odds ratio (OR) and 95\% confidence intervals being estimated at 1.006 (1.004-1.008), translating into 80 years-old respondents being $\sim 10 \%$ more likely to select the correct answer than 20 years-old respondents (Supplementary Figure 18). This trend was preserved when dealing with age as a 3-level factor (Table 2 and Figure 1). Knowledge also increased with increasing economic level, with the wealthiest people being 53\% more likely to provide the correct answer than less wealthy people (Table 2 and Supplementary Figure 19). Moreover, the odds of respondents who exhibited high affinity for nature or positive prejudice toward sharks replying correctly to knowledge questions were $60 \%$ higher than the ones of respondents with low affinity or negative prejudice (Table 2 and Figure 1). In fact, a negative prejudice counteracted the positive effects of affinity for nature upon knowledge about sharks which were observed in respondents with neutral or positive prejudice (Supplementary Figure 20). Despite little geographic variability, the Northeast region exhibited the lowest level of knowledge (Table 2 and Supplementary Figure 21).

\section{Perceptions Toward Sharks}

The three sampling treatments showed substantial variability in perceptions toward sharks. FEN tourists exhibited more positive perceptions than FEN residents and respondents from Recife, whose perceptions were similar (Table 2 and Figure 3 ). Notably, a positive effect of education level upon perceptions, which involved a $165 \%$ increase in the odds of respondents exhibiting more positive perceptions from elementary through superior education (Table 2 and Figure 3), was more striking in REC than in FEN treatments (Supplementary Figure 22). In contrast, positive effects of both affinity for nature and specific knowledge about sharks upon respondent perceptions (Table 2 and Figure 3) were observed in the two FEN treatments but not in REC (Supplementary Figures 23, 24). Prejudice toward sharks also influenced perceptions, with the probability of respondents exhibiting the most positive perceptions increasing from 40 to $62 \%$ when prejudice shifted from negative to positive (Table 2 and Figure 3). Yet, such an effect tended to be smaller in REC than in FEN treatments (Supplementary Figure 25).

Despite sampling treatment exhibiting such a diverse influence on perceptions toward sharks, the model selection procedure indicated that they were best predicted by the interaction of specific knowledge about sharks and education 
TABLE 2 | Ordinal logistic regression model results for the effects of single predictor variables on public knowledge about and perceptions toward sharks assessed with Likert questions.

\begin{tabular}{|c|c|c|c|c|c|c|c|c|c|c|}
\hline Response & Predictor & Reference & Levels & Coef & $S E$ & $t$-value & $p$-value & OR & $\mathrm{Cl}_{2.5 \%}$ & $\mathrm{Cl}_{97.5 \%}$ \\
\hline \multirow[t]{19}{*}{ Knowledge } & Affinity for nature & Low & Medium & 0.225 & 0.027 & 8.270 & $<0.001$ & 1.252 & 1.187 & 1.321 \\
\hline & & & High & 0.469 & 0.043 & 10.962 & $<0.001$ & 1.600 & 1.471 & 1.739 \\
\hline & Age & $<30$ years & $30-44$ years & 0.135 & 0.027 & 5.063 & $<0.001$ & 1.145 & 1.086 & 1.207 \\
\hline & & & $>44$ years & 0.192 & 0.033 & 5.744 & $<0.001$ & 1.212 & 1.135 & 1.294 \\
\hline & Economic level & Low & Medium & 0.121 & 0.033 & 3.689 & $<0.001$ & 1.129 & 1.059 & 1.204 \\
\hline & & & High & 0.425 & 0.078 & 5.458 & $<0.001$ & 1.530 & 1.315 & 1.785 \\
\hline & Education level & Elementary & High & 0.216 & 0.080 & 2.720 & 0.007 & 1.241 & 1.062 & 1.450 \\
\hline & & & Superior & 0.417 & 0.076 & 5.470 & $<0.001$ & 1.518 & 1.306 & 1.762 \\
\hline & Ocean proximity & Interior & Littoral & -0.013 & 0.043 & -0.295 & 0.768 & 0.988 & 0.908 & 1.074 \\
\hline & Prejudice toward sharks & Neutral & Negative & -0.254 & 0.038 & -6.635 & $<0.001$ & 0.775 & 0.719 & 0.836 \\
\hline & & & Positive & 0.254 & 0.027 & 9.454 & $<0.001$ & 1.289 & 1.223 & 1.359 \\
\hline & Region & Northeast & Center-West & 0.140 & 0.061 & 2.288 & 0.022 & 1.151 & 1.021 & 1.298 \\
\hline & & & South & 0.258 & 0.047 & 5.468 & $<0.001$ & 1.295 & 1.181 & 1.421 \\
\hline & & & Southeast & 0.284 & 0.027 & 10.283 & $<0.001$ & 1.329 & 1.259 & 1.403 \\
\hline & Sex & Female & Male & 0.015 & 0.024 & 0.641 & 0.521 & 1.015 & 0.969 & 1.064 \\
\hline & Specific knowledge & Low & Medium & 0.113 & 0.030 & 3.786 & $<0.001$ & 1.120 & 1.056 & 1.187 \\
\hline & & & High & 0.251 & 0.038 & 6.537 & $<0.001$ & 1.285 & 1.192 & 1.386 \\
\hline & Treatment & FENres & FENtour & 0.172 & 0.038 & 4.558 & $<0.001$ & 1.188 & 1.103 & 1.279 \\
\hline & & & REC & -0.194 & 0.040 & -4.877 & $<0.001$ & 0.823 & 0.762 & 0.890 \\
\hline \multirow[t]{19}{*}{ Perceptions } & Affinity for nature & Low & Medium & 0.283 & 0.033 & 8.527 & $<0.001$ & 1.327 & 1.244 & 1.416 \\
\hline & & & High & 0.585 & 0.052 & 11.227 & $<0.001$ & 1.794 & 1.621 & 1.988 \\
\hline & Age & $<30$ years & 30-44 years & 0.025 & 0.033 & 0.754 & 0.451 & 1.025 & 0.961 & 1.093 \\
\hline & & & $>44$ years & -0.113 & 0.040 & -2.807 & 0.005 & 0.894 & 0.826 & 0.967 \\
\hline & Economic level & Low & Medium & 0.135 & 0.043 & 3.146 & 0.002 & 1.144 & 1.052 & 1.244 \\
\hline & & & High & 0.441 & 0.102 & 4.343 & $<0.001$ & 1.554 & 1.277 & 1.901 \\
\hline & Education level & Elementary & High & 0.429 & 0.096 & 4.478 & $<0.001$ & 1.536 & 1.273 & 1.853 \\
\hline & & & Superior & 1.007 & 0.092 & 10.930 & $<0.001$ & 2.737 & 2.285 & 3.279 \\
\hline & Ocean proximity & Interior & Littoral & -0.260 & 0.052 & -4.964 & $<0.001$ & 0.771 & 0.695 & 0.854 \\
\hline & Prejudice toward sharks & Neutral & Negative & -0.431 & 0.047 & -9.266 & $<0.001$ & 0.650 & 0.593 & 0.712 \\
\hline & & & Positive & 0.431 & 0.033 & 13.055 & $<0.001$ & 1.539 & 1.442 & 1.641 \\
\hline & Region & Northeast & Center-West & 0.464 & 0.076 & 6.098 & $<0.001$ & 1.591 & 1.372 & 1.849 \\
\hline & & & $s$ & 0.433 & 0.058 & 7.465 & $<0.001$ & 1.542 & 1.377 & 1.729 \\
\hline & & & SE & 0.461 & 0.034 & 13.650 & $<0.001$ & 1.585 & 1.484 & 1.694 \\
\hline & Sex & Female & Male & 0.160 & 0.029 & 5.576 & $<0.001$ & 1.174 & 1.110 & 1.242 \\
\hline & Specific knowledge & Low & Medium & 0.222 & 0.036 & 6.154 & $<0.001$ & 1.249 & 1.164 & 1.340 \\
\hline & & & High & 0.398 & 0.047 & 8.524 & $<0.001$ & 1.489 & 1.359 & 1.632 \\
\hline & Treatment & FENres & FENtour & 0.545 & 0.045 & 12.223 & $<0.001$ & 1.727 & 1.582 & 1.885 \\
\hline & & & REC & 0.043 & 0.047 & 0.914 & 0.361 & 1.044 & 0.952 & 1.146 \\
\hline
\end{tabular}

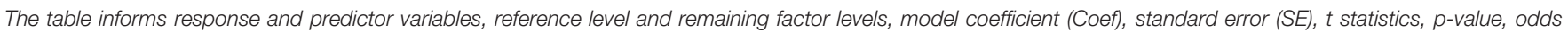
ratio $(\mathrm{OR})$ and lower $\left(\mathrm{Cl}_{2.5 \%}\right)$ and upper $\left(\mathrm{Cl}_{97.5 \%}\right)$ limits for OR 95\% confidence interval. Statistically significant results ( $\left.p<0.05\right)$ are highlighted in bold.

level added to respondent's sex. Males exhibited more positive perceptions than females (Table 3 and Supplementary Figure 26) most noticeably at the elementary education level (Supplementary Figure 27). Also, people with high specific knowledge about sharks were $45 \%$ more likely to exhibit positive perceptions than people with low specific knowledge (Table 2 and Figure 3), but such a trend turned out to be exclusive to people with elementary education. At this education level, a striking, threefold increase in the probability of respondents exhibiting more positive perceptions toward sharks was evidenced after their specific knowledge about sharks increased from medium to high (Table 3 and Figure 4). Perceptions at upper education levels showed to be unaffected or slightly influenced by specific knowledge.

Other relevant relationships were detected with singlepredictor models. Perceptions tended to become more negative with increasing age (Supplementary Figure 18) particularly among people with elementary education, contrasting with people with high or superior education for whom perceptions exhibited little variation through age (Supplementary Figure 28). Such an effect of age was indiscernible among FEN residents, though (Supplementary Figure 29). Likewise, perceptions 

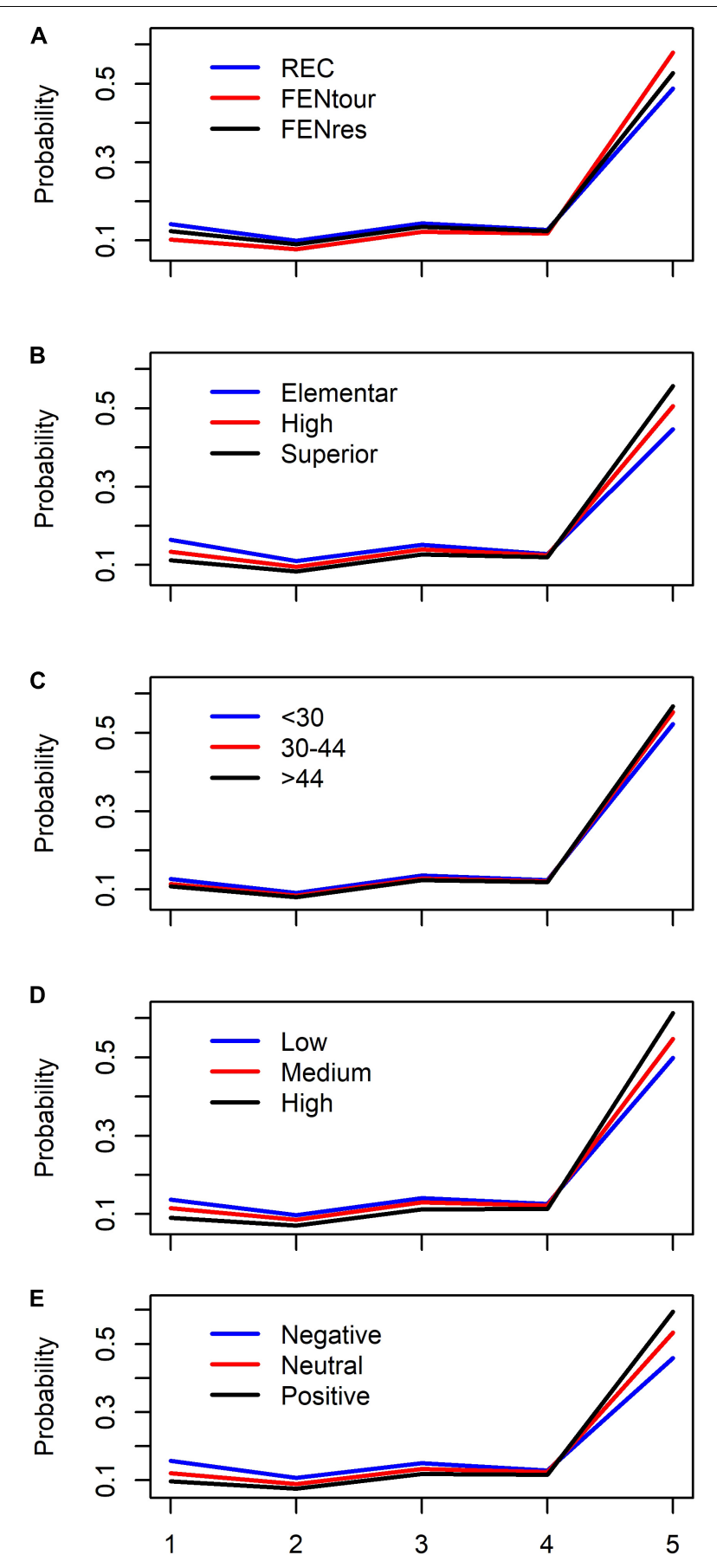

Likert scale

FIGURE 1 | Probability distribution of a 5-level Likert scale of knowledge about sharks across (A) sampling treatments (i.e., Recife - REC, tourists of Fernando de Noronha - FENtour, and residents at Fernando de Noronha FENres), (B) education level (i.e., elementary, high and superior education), (C) age classes (i.e., <30, 30-44, and $>44$ years old), (D) affinity for nature classes (i.e., low, medium, and high affinity for nature), and (E) types of prejudice toward sharks (i.e., negative, neutral, and positive prejudice), assessed with a Likert-based ordered logistic regression model. The Likert scale spans from 1 through 5 , with 5 being the most positive/correct option. Confidence intervals were not included for clarity sake. deteriorated with age among people with negative or neutral prejudice toward sharks, but they tended to become slightly more positive with increasing age when prejudice was positive (Supplementary Figure 30). Education level had a positive effect on perceptions only in people ascribed with either negative or neutral prejudice toward sharks (Supplementary Figure 31) and with either low or medium specific knowledge about sharks (Supplementary Figure 32), with respondents ascribed with positive prejudice and high specific knowledge exhibiting generally positive perceptions toward sharks regardless of their education level. In turn, a higher economic level translated into more positive perceptions toward sharks (Table 2 and Supplementary Figure 19). Curiously, an increased affinity for nature had a negative effect on perceptions among people with negative prejudice toward sharks, despite its effect was positive concerning people ascribed with neutral or positive prejudice (Supplementary Figure 33). Moreover, positive effects of respondents' affinity for nature on their perceptions tended to be greater with increased specific knowledge about sharks (Supplementary Figure 34). Finally, geographic differences in perceptions were conspicuous as inhabitants from the Northeast region exhibited much poorer perceptions than inhabitants from all other Brazilian regions, whose perceptions tended to be similar (Table 2 and Supplementary Figure 21).

\section{Relationship Between Knowledge and Perceptions}

Perceptions toward sharks correlated significantly with knowledge about sharks. Pearson's product-moment correlation indicated a direct proportionality between the two variables $(t=13.304 ; \mathrm{df}=1092 ; r=0.373 ; 95 \%$ confidence interval for $r=0.321-0.423 ; p<0.001)$. Complementary linear regression analysis sustained that respondent's Likert score for perceptions was directly proportional to the score for knowledge (intercept $=1.498$; slope $=0.626 ; p<0.001$ ), albeit model fit was low $\left(R^{2}=0.140\right)$ (Figure 5). An examination of correlation coefficients per sampling treatment demonstrated that the proportionality between perceptions and knowledge was preserved in all treatments, with Pearson's $r$ ranging from 0.293 in treatment FENtour to 0.353 in treatment REC.

\section{DISCUSSION}

A most relevant relationship between education level, knowledge about and perceptions toward sharks was evidenced in this study, with increased education and knowledge generally benefiting more positive perceptions. Previous research also associated higher education level with greater knowledge about sharks and more positive perceptions and attitudes toward these species (Thompson and Mintzes, 2002; O’Bryhim and Parsons, 2015; Tsoi et al., 2016; Lama et al., 2018). Misperceptions may derive from a lack of acquaintance with and understanding about sharks, rendering people susceptible to misinformation (Muter et al., 2012; Myrick and Evans, 2014). For example, the use of ominous soundtracks in shark documentaries exacerbates negative sentiments and perceptions of sharks among the audience (Nosal et al., 2016), but viewers who are aware of 
TABLE 3 | Ordinal logistic regression model results for the effects of multiple predictor variables on public knowledge about and perceptions toward sharks assessed with Likert questions.

\begin{tabular}{|c|c|c|c|c|c|c|c|c|c|}
\hline Response & Predictor level & Interaction term & Coef & $S E$ & $t$-value & $p$-value & OR & $\mathrm{Cl}_{2.5 \%}$ & $\mathrm{Cl}_{97.5 \%}$ \\
\hline \multirow[t]{9}{*}{ Knowledge $^{\dagger}$} & Specific knowledge medium & & 0.325 & 0.086 & 3.791 & $<0.001$ & 1.385 & 1.170 & 1.638 \\
\hline & Specific knowledge high & & 0.522 & 0.107 & 4.860 & $<0.001$ & 1.685 & 1.366 & 2.080 \\
\hline & Treatment FENtour & & 0.362 & 0.079 & 4.604 & $<0.001$ & 1.437 & 1.231 & 1.676 \\
\hline & Treatment REC & & 0.143 & 0.085 & 1.677 & 0.093 & 1.153 & 0.976 & 1.362 \\
\hline & Ocean proximity littoral & & 0.120 & 0.047 & 2.560 & 0.011 & 1.127 & 1.028 & 1.235 \\
\hline & Specific knowledge medium & Treatment FENtour & -0.176 & 0.096 & -1.838 & 0.066 & 0.839 & 0.696 & 1.012 \\
\hline & Specific knowledge high & Treatment FENtour & -0.190 & 0.120 & -1.587 & 0.113 & 0.827 & 0.654 & 1.045 \\
\hline & Specific knowledge medium & Treatment REC & -0.336 & 0.102 & -3.293 & $<0.001$ & 0.715 & 0.585 & 0.873 \\
\hline & Specific knowledge high & Treatment REC & -0.574 & 0.130 & -4.429 & $<0.001$ & 0.563 & 0.435 & 0.726 \\
\hline \multirow[t]{9}{*}{ Perceptions ${ }^{\ddagger}$} & Specific knowledge medium & & 0.093 & 0.205 & 0.456 & 0.658 & 1.098 & 0.735 & 1.641 \\
\hline & Specific knowledge high & & 2.201 & 0.390 & 5.641 & $<0.001$ & 9.029 & 4.347 & 20.328 \\
\hline & Education level high & & 0.571 & 0.171 & 3.344 & $<0.001$ & 1.769 & 1.266 & 2.474 \\
\hline & Education level superior & & 1.116 & 0.163 & 6.851 & $<0.001$ & 3.054 & 2.219 & 4.205 \\
\hline & Sex male & & 0.145 & 0.031 & 4.670 & $<0.001$ & 1.155 & 1.088 & 1.228 \\
\hline & Specific knowledge medium & Education level high & 0.021 & 0.219 & 0.097 & 0.923 & 1.022 & 0.665 & 1.570 \\
\hline & Specific knowledge high & Education level high & -2.138 & 0.404 & -5.294 & $<0.001$ & 0.118 & 0.051 & 0.252 \\
\hline & Specific knowledge medium & Education level superior & 0.042 & 0.209 & 0.199 & 0.843 & 1.043 & 0.691 & 1.572 \\
\hline & Specific knowledge high & Education level superior & -1.869 & 0.394 & -4.743 & $<0.001$ & 0.154 & 0.068 & 0.323 \\
\hline
\end{tabular}

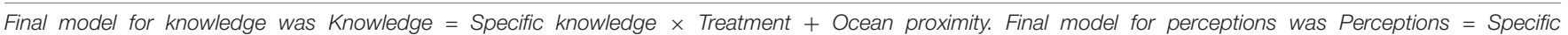

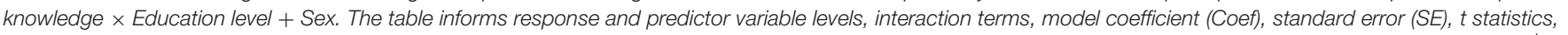

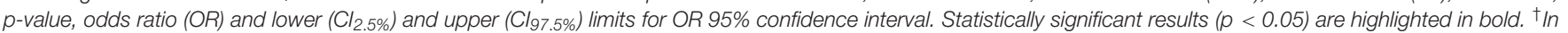

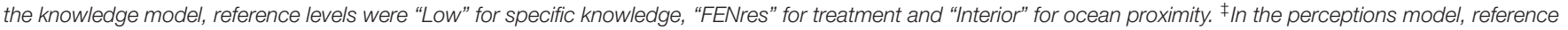
levels were "Low" for specific knowledge, "Elementary" for education level and "Female" for sex.

the manipulative purpose of such a soundtrack might not be influenced by it. Investing on educational development could thus translate into improved public knowledge about sharks, more favorable perceptions regarding their value, and widened support for shark conservation. In this regard, environmental education has proven successful in raising public awareness and support for the protection of potentially hazardous aquatic predators (Ploeg et al., 2011) and it should be used extensively as a shark conservation instrument. Furthermore, OLR models indicated that an increased specific knowledge about sharks would translate into a dramatic improvement in the perceptions of elementary-educated people toward these species. This suggests that shark-specific educative action could provide a cognitive shortcut to develop affective feelings toward sharks and promote conservation support, as observed by Curtin and Papworth (2018). Raising the education level of a whole population to collect conservation benefits is a colossal endeavor which might fail to provide successful outcomes in due time, particularly in less developed countries. Increasing citizen's knowledge specifically about sharks could thus provide a more immediate, cost-effective strategy to combat negative prejudice toward sharks and promote better perceptions about these species. Albeit the overall quality of knowledge about and perceptions toward sharks assessed in this survey was high, it should be noted that the response scale used is largely dependent on the assertions posed by the questionnaire because different assertions could have led to different frequency distributions across the five Likert options. Notwithstanding, we were seeking to capture the relative dynamics in knowledge and perceptions across a multitude of factors, therefore the absolute magnitude of the global response was of least importance to our goals.

The effects ascribed to sampling treatment revealed some interesting clues about the differences in knowledge and perception quality to be expected before opposite shark-related scenarios. First, knowledge about sharks was greater in both FEN treatments even though many respondents from REC held university degrees. This difference may relate to FEN respondents being ecotourists or residing in an MPA, which can increase environmental knowledge and support for conservation (Powell and Ham, 2008). Second, perceptions toward sharks were much less positive among Pernambuco residents (i.e., treatments REC and FENres) compared to FEN tourists, a trend likely derived from the shark hazard problem in this state. Low perception scores by FEN residents were unexpected but may derive from many of these people being migrant, low-educated workers coming from Pernambuco's mainland. Garla et al. (2015) also reported FEN tourists to have greater knowledge of and more positive attitudes toward sharks than FEN residents, but the opposite trend was observed in the Galapagos Archipelago (Acuña-Marrero et al., 2018) where shark peril is not an issue. Although people's attitudes regarding shark hazard could currently be less focused on human welfare and more on wildlife (Neff and Yang, 2013; Pepin-Neff and Wynter, 2018b), shark bite incidents may still generate considerable commotion and deteriorate public perceptions toward the putative menace posed by these predators (Pepin-Neff and Wynter, 2018a), even in countries with no records of such incidents (Lama et al., 2018). Furthermore, since sharks do not elicit fright responses in young 


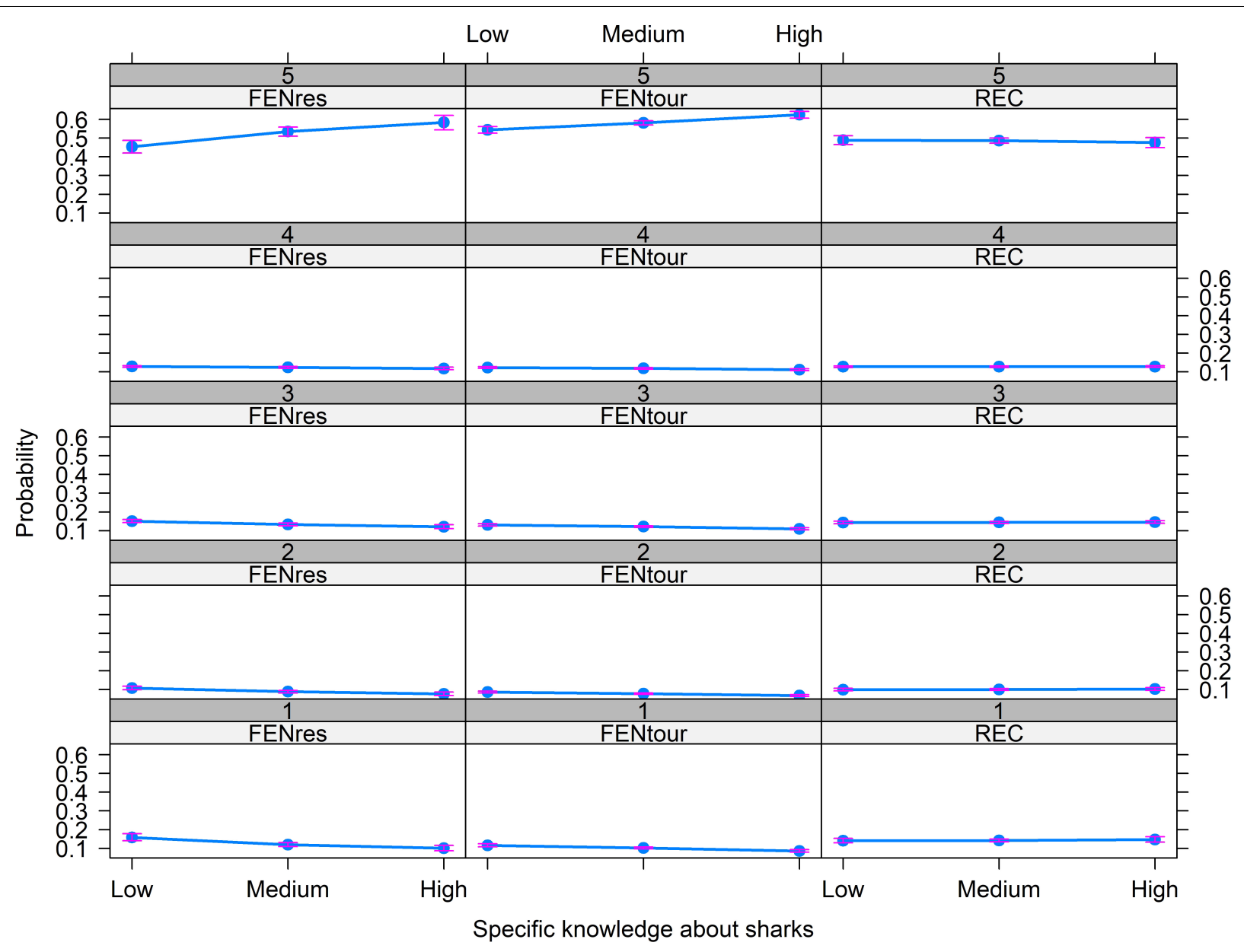

FIGURE 2 | Effects of the interaction of sampling treatment (i.e., Recife - REC, tourists of Fernando de Noronha - FENtour, and residents at Fernando de Noronha FENres) with specific knowledge about sharks (i.e., low, medium, and high) on the variability in knowledge about sharks, assessed with a Likert-based ordered logistic regression model. The Likert scale spans from 1 through 5 , with 5 being the most positive/correct option. The vertical axes represent the probability of respondents selecting each of the Likert options. The three panel columns correspond to sampling treatments (FENres, FENtour, and REC from the left through the right columns), whereas the five panel rows correspond to Likert options (ordered in descending order from the top through the bottom rows). Note that specific knowledge about sharks is a predictor variable derived with questions on specific quantitative metrics about sharks, whilst knowledge about sharks is a response variable derived with Likert questions about more general aspects of sharks. The complete model also included ocean proximity as a predictor variable.

children (Rakison, 2018), it seems likely that negative perceptions toward shark peril are acquired later in the cognitive development process rather than being intrinsic to the human being. Again, the role of news media and audiovisual entertainment in disseminating disproportionately negative perceptions toward sharks could be determinative (McCagh et al., 2015; Sebatier and Huveneers, 2018), since most people are not expected to have any direct or indirect involvement with sharks.

Yet, inhabitants from Pernambuco are not only more involved with shark-human conflicts but they are also more exposed to negative media coverage of sharks than inhabitants from other Brazilian states, which could lead to distinctive community responses and societal behaviors toward sharks. For example, a conspicuously positive influence of specific knowledge about sharks (i.e., acquaintance with quantitative metrics on sharkrelated subjects denoting an appreciation for sharks) and affinity for nature on Likert-derived knowledge and perception levels turned out to be canceled in treatment REC. This suggests that knowledge about and perceptions toward sharks in Recife are homogeneous regardless of people's attachment to nature and predisposition to learn more specific details about these animals. On the other hand, the positive effect of education level on perceptions toward sharks was more intense in REC, where respondents also exhibited a more negative prejudice toward sharks and a lower affinity for nature. The population from REC may be possibly overwhelmed by too much information and negativism toward shark peril after cohabiting with persistently dramatic shark bite incidents for several years, as suggested by the lower ratings in perceptions toward sharks. While engaging into positive experiences with sharks may contribute to enhance perceptions and promote support for their conservation (Friedrich et al., 2014; Skubel et al., 2019), the reverse may also stand generally true (but see Neff and Yang, 2013). Contrasting shark-related experiences, even if not lived in 

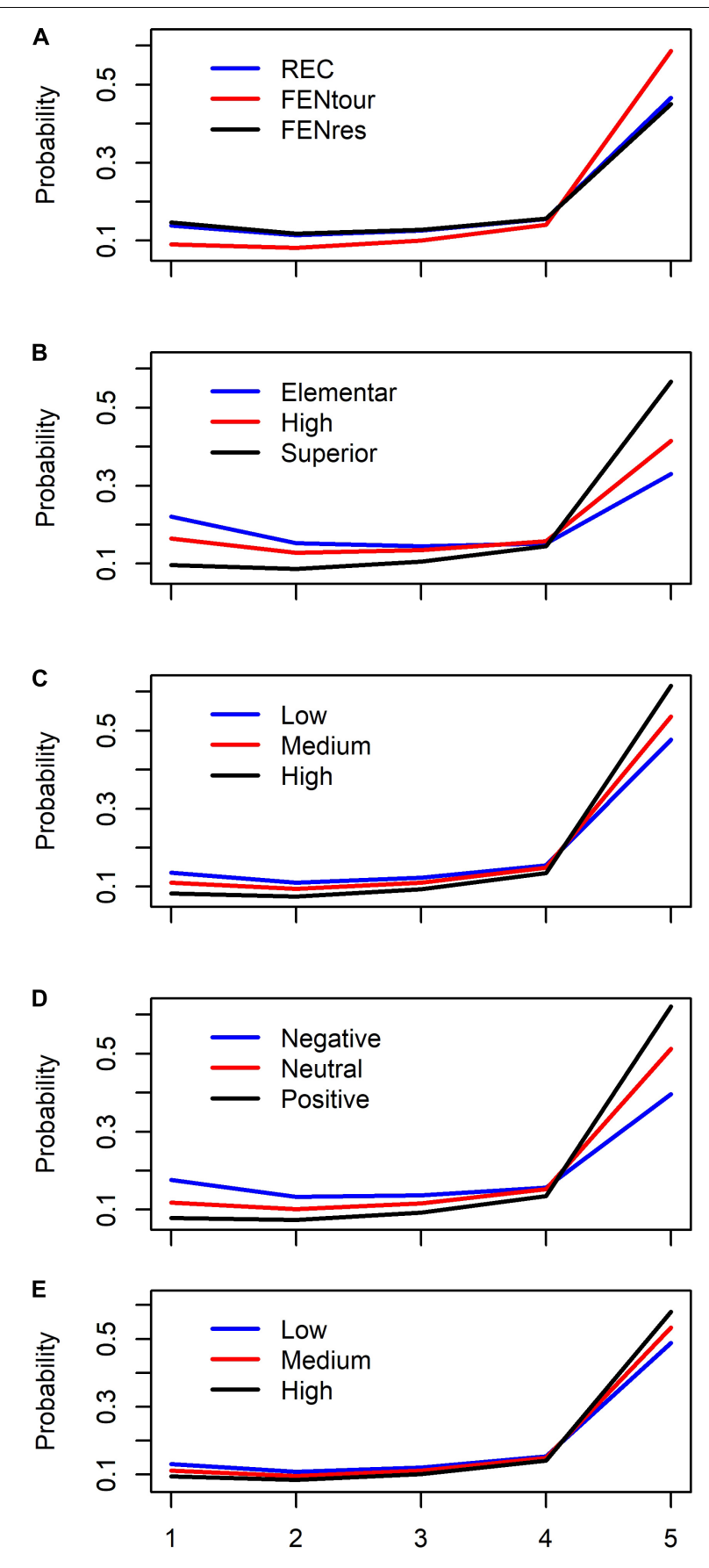

Likert scale

FIGURE 3 | Probability distribution of a 5-level Likert scale of perceptions toward sharks across (A) sampling treatments (i.e., Recife - REC, tourists of Fernando de Noronha - FENtour, and residents at Fernando de Noronha FENres), (B) education level (i.e., elementary, high, and superior education), (C) affinity for nature classes (i.e., low, medium, and high affinity for nature), (D) types of prejudice toward sharks (i.e., negative, neutral, and positive prejudice), and (E) classes of specific knowledge about sharks (i.e., low, medium, and high specific knowledge), assessed with a Likert-based ordered logistic regression model. The Likert scale spans from 1 through 5 , with 5 being the most positive/correct option. Confidence intervals were not included for clarity sake. person, should be partially shaping the disparities observed between Fernando de Noronha and Recife. Nonetheless, people inhabiting shark hazardous areas seem to respond positively to educational processes, endowing managers with an opportunity to ameliorate perceptions among these communities. Specific outreach measures which take the particularities of such communities into account may be required, though, since the influence of some relevant predictors at REC shifted in magnitude and directionality compared to FEN.

A suite of demographic, economic and socioenvironmental variables influenced the quality in public shark-related knowledge and perceptions assessed in this study. Such information is potentially useful to conservation management by identifying high-priority segments of the society that warrant particular attention and by providing a guideline for tailoring customized strategies to boost public awareness and support for conservation, since different audiences may be best persuaded by distinct approaches (Ashley et al., 2019). For example, perceptions toward sharks seem to be worst among older and less wealthy people and among low-educated females. Hence, prioritizing cognitive and emotional enhancement policies targeted to $>44$ years old citizens, women with elementary education and lowincome people might prove advantageous. Females and older respondents also exhibited more negative attitudes toward sharks in Galapagos (Acuña-Marrero et al., 2018), whereas diminished environmental concern has been previously associated with economic difficulties (Scruggs and Benegal, 2012). In contrast, positive effects of age on student environmental awareness and attitude have been detected (Aminrad et al., 2011). Also, a comprehensive meta-analysis reported negligible effects of age on pro-environmental behaviors albeit older individuals tending to exhibit a higher affinity for nature (Wiernik et al., 2013), similarly to the trend described in this study. The specificities of sharks as a survey topic which possibly pleases young people the most might explain the observed effects of age on perception variability. Nonetheless, it was verified that a low education level exacerbated the negative effect of age on perceptions whereas a positive prejudice toward sharks overruled such an effect and actually promoted better perceptions with increasing age. Such an observation adds to previous evidence that perceptions toward sharks could be consistently improved by fostering cognitive and emotional development focused on ocean literacy, conservation awareness, and nature experiencing.

Prejudice may, in fact, be a limiting factor concerning the improvement of public knowledge about and perceptions toward sharks since the positive effects ascribed to affinity for nature and to specific knowledge about sharks were much reduced or absent when prejudice was negative. Also, education level had little contribution to perception improvement among people with positive prejudice because this group generally exhibited good knowledge and positive perceptions toward sharks. These trends sustain the leverage of the emotional component on the regulation of human attitudes toward sharks (Tsoi et al., 2016; Lama et al., 2018) and suggest that considerable improvements in perceptions could be achieved by resolving the prevalence of unjustified feelings and prejudice resulting from societal misconceptions about these species. Augmenting the specific 


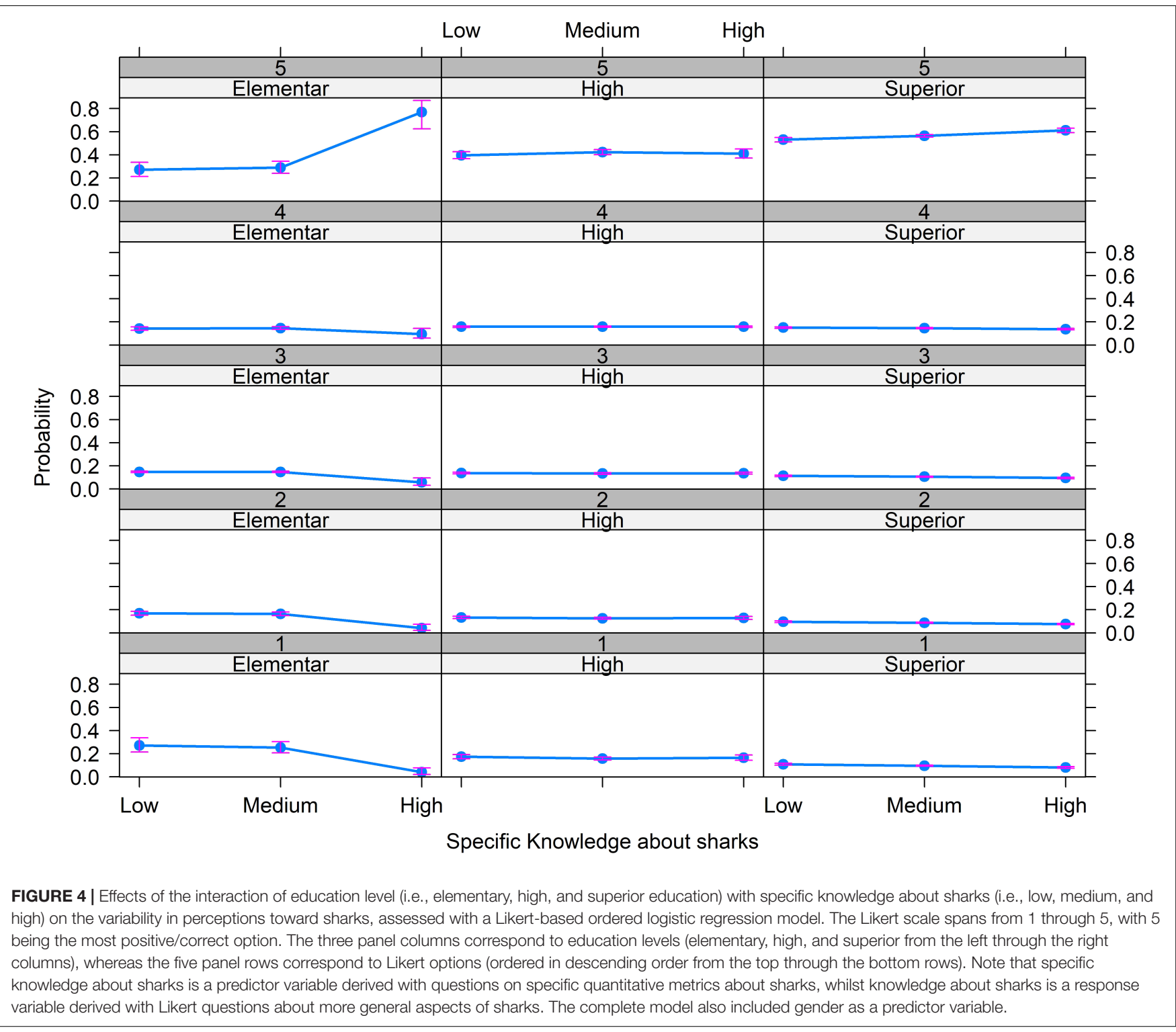

knowledge about sharks and promoting a higher affinity for nature among citizens could stem as an efficient contribution toward such a goal, although such a strategy may prove ineffective among populations exposed to persistent shark peril.

Building positive environmental attitudes and conservation awareness is an intricate, multidimensional task that requires extensive use of effective social engineering tools in combination with environmental and educational sciences. However, such efforts must be conducted despite their magnitude because the accomplishments of conservation action are greatly influenced by human attitudes and behaviors (Veríssimo et al., 2012). For example, the current level of fishing pressure on oceanic sharks could be partially ascribed to the marginalization of these taxa by managers, stakeholders, and societies in general, which may have impeded the timely implementation of effective conservation policies (Jacques, 2010). On that account, global awareness and support for shark conservation has been advocated by a growing body of concerned researchers, stakeholders, and organizations. All these efforts might translate into a greater exposure of the general public to this subject and potentially contribute to enhance the effectiveness of conservation policies to some extent. Yet, the modern world is being quickly introduced to great anthropogenic environmental threats, such as global warming and plastic pollution, which are absorbing much of the attention of societies. Engaging the public with specific environmental causes might thus become a competitive venue which will require comprehensive outreach measures and the cooperation of conservation actors. At this point, however, disparities in perceptions about the risk and consequences of shark collapse are readily observable even among shark experts, whose opinions regarding the conservation status of sharks and the sustainability of their fisheries seem to diverge (Braccini, 2016). Endeavoring to enhance and harmonize awareness toward shark sustainability throughout the society should be seriously considered. 


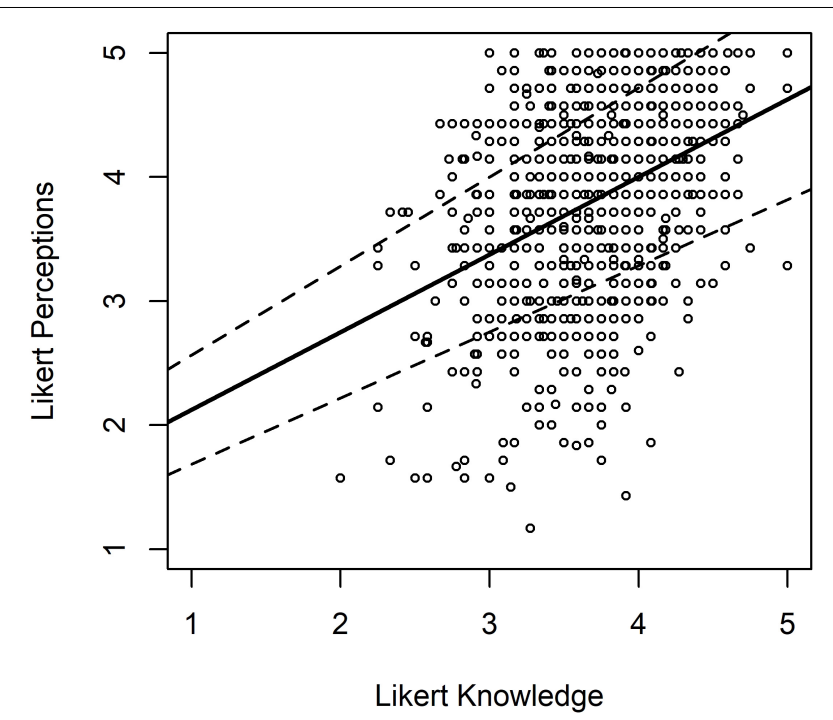

FIGURE 5 | Linear regression model of respondent's knowledge about sharks against respondent's perceptions toward sharks assessed with 5-level Likert data. The solid line represents the model estimate, the dashed lines represent 95\% confidence intervals and blank circles represent the empirical data. Coefficients for intercept and slope were 1.498 and 0.626 , respectively.

Raising environmental awareness and ocean literacy among societies will be instrumental for producing behavioral changes toward more sustainable, ecofriendly lifestyles (United Nations, 2018) and toward a more active participation of the society in conservation action. The urgency to achieve specific conservation goals for alleviating human pressure on endangered shark species warrants the implementation of dedicated outreach mechanisms based on the resources of social disciplines, such as marketing techniques (Wright et al., 2015), and fueled by expanded knowledge about the several aspects of the human-shark relationship. It is known that public engagement in conservation action and pro-environmental behavior may be influenced by a plethora of intrinsic and extrinsic factors (Kollmuss and Agyeman, 2002). Our results contribute to a better understanding of the effects of some relevant predictors of shark-related knowledge and perceptions across distinct socioenvironmental scenarios, besides providing essential information for the tailored optimization of these societal traits to garner support for shark conservation and promote ocean citizenship (Fletcher and Potts, 2007). Our interpretations were generally compatible with previously published research; yet, the direct applicability of this study to other regions might require caution as geographical variability in knowledge and perception responses likely regulated by region-specific parameters was detected. Anyhow, a notable coherence between the trends predicted by socioenvironmental proxies and the expected results indicates that these proxies were adequately configured and that they effectively represent the socioenvironmental values for which they were designated. Such proxies proved to be useful in capturing some of the human facets associated with awareness and discernment variability on the topics of sharks and their use should be considered when surveying public responses regarding wildlife. Further research dedicated to the multiple dimensions of cognitive and emotional processes involving sharks is needed to understand the human component of shark conservation more thoroughly. Meanwhile, a substantial amount of data about the factors modulating societal attitudes and support toward sharks is at disposal of managers and stakeholders for being incorporated into smart, human-oriented conservation policies, which are utterly required in face of the ongoing anthropogenic threats to shark populations and to the environment as a whole.

\section{CONCLUSION}

Understanding the sources of variability in public knowledge about and perceptions toward sharks enables the development of tailored strategies to raise crucial awareness and support for shark conservation. Conspicuous linkages between the cognitive (i.e., knowledge/acquaintance) and affective (i.e., perceptions/feelings) domains detected by this study suggest that lack of knowledge may lead to impoverished perceptions, besides indicating that perception improvement could be achieved through educational processes combined with nature experiencing promotion. Although increasing citizen's education level is a societal goal in any circumstance, more immediate dissemination of sharkspecific knowledge could boost perceptions and attitudes toward the conservation of these species in a timely, cost-effective fashion. Besides education, managers should also focus on addressing unjustified negative prejudice toward sharks since this trait may override potentially positive effects of other human attributes and compromise the effectiveness of conservation action. Other socioeconomic factors which could be practical for optimizing socioenvironmental gains against public engagement efforts include age, economic level, and gender. Moreover, populations exposed to shark peril, such as the one at Recife, may require specific outreach measures because some of the factors analyzed revealed structural differences in their effects when compared to the population sampled in the marine protected area. The results herein reported, together with previously published research on this subject, could endow managers with a consubstantiated framework to deal with the human dimensions of shark conservation. As anthropogenic pressure continues to threaten whole ecosystems and the general biodiversity in current times, ensuring that societies comply with sustainable and pro-environmental behaviors toward species conservation has become imperative.

\section{DATA AVAILABILITY STATEMENT}

All datasets generated for this study are included in the article/Supplementary Material.

\section{ETHICS STATEMENT}

The studies involving human participants were reviewed and approved by Committee for Ethics in Research with 
Human Beings of the Federal University of Pernambuco. The patients/participants provided their written informed consent to participate in this study.

\section{AUTHOR CONTRIBUTIONS}

AA conceived and designed the study. PR, LF, and LV conducted data sampling. AA performed data analysis with contributions from AC, PM, and SL. AA wrote the manuscript with contributions from all authors. FH supervised the execution of the project.

\section{FUNDING}

This research was funded by the National Geographic Society Conservation Trust (ref. \#C314-15), Fundação para a Ciência e Tecnologia -FCT (ref. UID/MAR/04292/2013), and the Integrated Programme of SR\&TD "Smart Valorization of Endogenous Marine Biological Resources Under a Changing

\section{REFERENCES}

Acuña-Marrero, D., Cruz-Modino, R., Smith, A. N. H., Salinas-de-León, P., Pawley, M. D. M., and Anderson, M. J. (2018). Understanding human attitudes towards sharks to promote sustainable coexistence. Mar. Pol. 91, 122-128. doi: 10.1016/ j.marpol.2018.02.018

Afonso, A. S., Andrade, H. A., and Hazin, F. H. V. (2014). Structure and dynamics of the shark assemblage off recife, Northeastern Brazil. PLoS One 9:e102369. doi: 10.1371/journal.pone.0102369

Afonso, A. S., Cantareli, C. V., Levy, R. P., and Veras, L. B. (2016). Evasive mating behaviour by female nurse sharks, Ginglymostoma cirratum (Bonnaterre, 1788), in an equatorial insular breeding ground. Neotrop. Ichthyol. 14:e160103.

Afonso, A. S., Fidelis, L., Roque, P., Galindo, R., Dionisio, W., Veras, L., et al. (2019). Public support for conservation may decay with increasing residence time in suboptimal marine protected areas. Mar. Pol. 108:103665. doi: 10.1016/ j.marpol.2019.103665

Afonso, A. S., Garla, R., and Hazin, F. H. V. (2017b). Tiger sharks can connect equatorial habitats and fisheries across the Atlantic Ocean basin. PLoS One 12:e0184763. doi: 10.1371/journal.pone.0184763

Afonso, A. S., Niella, Y. V., and Hazin, F. H. V. (2017a). Inferring trends and linkages between shark abundance and shark bites on humans for shark-hazard mitigation. Mar. Freshw. Res. 68, 1354-1365.

Aminrad, Z., Zakaria, S. Z. B. S., and Hadi, A. S. (2011). Influence of age and level of education on environmental awareness and attitude: case study on Iranian students in Malaysian Universities. Soc. Sci. 6, 15-19. doi: 10.3923/sscience. 2011.15.19

Ashley, M., Pahl, S., Glegg, G., and Fletcher, S. (2019). A change of mind: applying social and behavioral research methods to the assessment of the effectiveness of ocean literacy initiatives. Front. Mar. Sci. 6:288. doi: 10.3389/fmars.2019. 00288

Barney, E. C., Mintzes, J. J., and Yen, C. F. (2005). Assessing knowledge, attitudes, and behavior toward charismatic megafauna: the case of dolphins. J. Environ. Educ. 36, 41-55. doi: 10.3200/joee.36.2.41-55

Bennett, N., and Dearden, P. (2014). Why local people do not support conservation: community perceptions of marine protected area livelihood impacts, governance and management in Thailand. Mar. Pol. 44, 107-116. doi: 10.1016/j.marpol.2013.08.017

Bögeholz, S. (2006). Nature experience and its importance for environmental knowledge, values and action: recent German empirical contributions. Environ. Educ. Res. 12, 65-84. doi: 10.1080/13504620500526529

Bond, M. E., Valentin-Albanese, J., Babcock, E. A., Heithaus, M. R., Grubbs, R. D., Cerrato, R., et al. (2019). Top predators induce habitat shifts in prey within
Climate" (ref. Centro-01-0145-FEDER-000018), co-funded by Centro 2020 program, Portugal 2020, and European Union through the European Regional Development Fund.

\section{ACKNOWLEDGMENTS}

We are grateful to Renato Galindo and Wanderson Dionisio for assisting with field work, and to the District Administration of Fernando de Noronha and to the Instituto Chico Mendes de Conservação da Biodiversidade (ICMBio) for providing tax exemptions, work permits and logistic support. We are also thankful for the valuable suggestions provided by two reviewers.

\section{SUPPLEMENTARY MATERIAL}

The Supplementary Material for this article can be found online at: https://www.frontiersin.org/articles/10.3389/fmars. 2020.00663/full\#supplementary-material

marine protected areas. Oecologia 190, 375-385. doi: 10.1007/s00442-01904421-0

Bornatowski, H., Navia, A. F., Braga, R. R., Abilhoa, V., and Corrêa, M. F. M. (2014). Ecological importance of sharks and rays in a structural foodweb analysis in southern Brazil. ICES J. Mar. Sci. 71, 1586-1592. doi: 10.1093/ icesjms/fsu025

Braccini, M. (2016). Experts have different perceptions of the management and conservation status of sharks. Ann. Mar. Biol. Res. 3, 1012.

Cardeñosa, D. (2019). Genetic identification of threatened shark species in pet food and beauty care products. Conserv. Genet. 20, 1383-1387. doi: 10.1007/s10592019-01221-0

Casey, A. L., Krausman, P. R., Shaw, W. W., and Shaw, H. G. (2005). Knowledge of and attitudes towards mountain lions: a public survey of residents adjacent to Saguaro National Park, Arizona. Hum. Dimens. Wildl. 10, 29-38. doi: 10.1080/ 10871200590904860

Casini, M., Hjelm, J., Molinero, J. C., Lovgren, J., Cardinale, M., Bartolino, V., et al. (2009). Trophic cascades promote threshold-like shifts in pelagic marine ecosystems. Proc. Natl. Acad. Sci. U.S.A. 106, 197-202. doi: 10.1073/pnas. 0806649105

CITES (2019). The CITES Appendices. Available online at https://www.cites.org/ eng/app/appendices.php (accessed November 11, 2019).

Cortés, E. (2000). Life history patterns and correlations in sharks. Rev. Fish. Sci. 8, 299-344. doi: 10.1080/10641260008951115

Curtin, P., and Papworth, S. (2018). Increased information and marketing to specific individuals could shift conservation support to less popular species. Mar. Pol. 88, 101-107. doi: 10.1016/j.marpol.2017.11.006

Davidson, L. N. K., Krawchuk, M. A., and Dulvy, N. K. (2015). Why have global shark and ray landings declined: improved management or overfishing? Fish Fish. 17, 438-458. doi: 10.1111/faf.12119

Dent, F., and Clarke, S. C. (2015). State of the Global Market for Shark Products. FAO Fisheries and Aquaculture Technical Paper No. 590. Rome: FAO, 187.

Dulvy, N. K., Baum, J. K., Clarke, S., Compagno, L. J. V., Cortés, E., Domingo, A., et al. (2008). You can swim but you can't hide: the global status and conservation of oceanic pelagic sharks and rays. Aquat. Conserv. Mar. Freshw. Ecosyst. 18, 459-482. doi: 10.1002/aqc. 975

Dulvy, N. K., Fowler, S. L., Musick, J. A., Cavanagh, E. D., Kyne, P. M., Harrison, L. R., et al. (2014). Extinction risk and conservation of the world's sharks and rays. eLife 3:e00590.

Fletcher, S., and Potts, J. (2007). Ocean citizenship: an emergent geographical concept. Coast. Manag. 35, 511-524. doi: 10.1080/08920750701525818

Fox, J., and Weisberg, S. (2019). An R Companion to Applied Regression, 3rd Edn. Thousand Oaks CA: Sage. 
Friedrich, L. A., Jefferson, R., and Glegg, G. (2014). Public perceptions of sharks: gathering support for shark conservation. Mar. Pol. 47, 1-7. doi: 10.1016/j. marpol.2014.02.003

Garla, R. C., Freitas, R. H. A., Calado, J. F., Paterno, G. B. C., and Carvalho, A. R. (2015). Public awareness of the economic potential and threats to sharks of a tropical oceanic archipelago in the western South Atlantic. Mar. Pol. 60, 128-133. doi: 10.1016/j.marpol.2015.06.012

Garla, R. C., Garcia, J., Veras, L. B., and Lopes, N. P. (2009). Fernando de Noronha as an insular nursery area for lemon sharks, Negaprion brevirostris, and nurse sharks, Ginglymostoma cirratum, in the equatorial western Atlantic Ocean. Mar. Biodivers. Rec. 2:e109. doi: 10.1017/S1755267209000670

Hazin, F. H. V., and Afonso, A. S. (2014). A green strategy for shark attack mitigation off Recife, Brazil. Anim. Conserv. 17, 287-296. doi: 10.1111/acv. 12096

Hazin, F. H. V., Burgess, G., and Carvalho, F. C. (2008). A shark attack outbreak off Recife, Pernambuco, Brazil: 1992-2006. Bull. Mar. Sci. 82, 199-212.

Heupel, M. R., Knip, D. M., Simpfendorfer, C. A., and Dulvy, N. K. (2014). Sizing up the ecological role of sharks as predators. Mar. Ecol. Prog. Ser. 495, 291-298. doi: 10.3354/meps 10597

Hussey, N. E., MacNeil, M. A., Siple, M. C., Popp, B. N., Dudley, S. F. J., and Fisk, A. T. (2015). Expanded trophic complexity among large sharks. Food Webs 4, 1-7. doi: 10.1016/j.fooweb.2015.04.002

Huveneers, C., Ebert, D. A., and Dudley, S. F. J. (2015). The evolution of chondrichthyan research through a metadata analysis of dedicated international conferences between 1991 and 2014. Afr. J. Mar. Sci. 37, 129-139. doi: 10.2989/ 1814232x.2015.1042911

ICCAT (2016). Report of the Inter-sessional Meeting of the Compliance Committee of the International Commission for the Conservation of Atlantic Tunas., Madrid, Spain, March 4-5. Available online at https://www.iccat.int/Documents/ Meetings/Docs/2016_FINAL_COC_REP_ENG.pdf (accessed November 11, 2019).

Jacques, P. J. (2010). The social oceanography of top oceanic predators and the decline of sharks: a call for a new field. Prog. Oceanog. 86, 192-203. doi: 10.1016/j.pocean.2010.04.001

Kollmuss, A., and Agyeman, J. (2002). Mind the Gap: why do people act environmentally and what are the barriers to pro-environmental behaviour? Environ. Educ. Res. 8, 239-260. doi: 10.1080/13504620220145401

Lama, L. R., De la Puente, S., and Riveros, J. C. (2018). Attitudes and misconceptions towards sharks and shark meat consumption along the Peruvian coast. PLoS One 13:e0202971. doi: 10.1371/journal.pone.020 2971

Leiserowitz, A. A., Kates, R. W., and Parris, T. M. (2005). Do global attitudes and behaviors support sustainable development? Environment 47, 22-38. doi: 10.3200/envt.47.9.22-38

McCagh, C., Sneddon, J., and Blanche, D. (2015). Killing sharks: the media's role in public and political response to fatal human-shark interactions. Mar. Pol. 62, 271-278. doi: 10.1016/j.marpol.2015.09.016

McCauley, D. J., Young, H. S., Dunbar, R. B., Estes, J. A., Semmens, B. X., and Micheli, F. (2012). Assessing the effects of large mobile predators on ecosystem connectivity. Ecol. Appl. 22, 1711-1717. doi: 10.1890/111653.1

Mitchell, J. D., McLean, D. L., Collin, S. P., and Langlois, T. L. (2018). Shark depredation in commercial and recreational fisheries. Rev. Fish Biol. Fisher. 28, 715-748. doi: 10.1007/s11160-018-9528-Z

Muter, B. A., Gore, M. L., Gledhill, K. S., Lamont, C., and Huveneers, C. (2012). Australian and U.S. news media portrayal of sharks and their conservation. Conserv. Biol. 27, 187-196. doi: 10.1111/j.1523-1739.2012.01 952.x

Myers, R. A., Baum, J. K., Shepherd, T. D., Powers, S. P., and Peterson, C. H. (2007). Cascading effects of the loss of apex predatory sharks from a coastal ocean. Science 315, 1846-1850. doi: 10.1126/science.1138657

Myrick, J. G., and Evans, S. D. (2014). Do PSAs take a bite out of shark week? The effects of juxtaposing environmental messages with violent images of shark attacks. Sci. Commun. 36, 544-569. doi: 10.1177/107554701454 7159

Neff, C. L., and Yang, J. Y. H. (2013). Shark bites and public attitudes: Policy implications from the first before and after shark bite survey. Mar. Pol. 38, 545-547. doi: 10.1016/j.marpol.2012.06.017
Nosal, A. P., Keenan, E. A., Hastings, P. A., and Gneezy, A. (2016). The effect of background music in shark documentaries onviewers' perceptions of sharks. PLoS One 11:e0159279. doi: 10.1371/journal.pone.0159279

O’Bryhim, J. R., and Parsons, E. C. M. (2015). Increased knowledge about sharks increases public concern about their conservation. Mar. Pol. 56, 43-47. doi: 10.1016/j.marpol.2015.02.007

Pepin-Neff, C., and Wynter, T. (2018b). Shark bites and shark conservation: an analysis of human attitudes following shark bite incidents in two locations in Australia. Conserv. Lett. 11, 1-8.

Pepin-Neff, C. L., and Wynter, T. (2018a). Reducing fear to influence policy preferences: An experiment with sharks and beach safety policy options. Mar. Pol. 88, 222-229. doi: 10.1016/j.marpol.2017.11.023

Ploeg, J., Cauilan-Cureg, M., Weerd, M., and De Groot, W. T. (2011). Assessing the effectiveness of environmental education: mobilizing public support for Philippine crocodile conservation. Conserv. Lett. 4, 313-323. doi: 10.1111/j. 1755-263x.2011.00181.x

Powell, R. B., and Ham, S. H. (2008). Can ecotourism interpretation really lead to pro-conservation knowledge, attitudes and behaviour? Evidence from the Galapagos Islands. J. Sustain. Tour. 16, 467-489. doi: 10.1080/ 09669580802154223

Queiroz, N., Humphries, N. E., Couto, A., Vedor, M., Costa, I., Sequeira, A. M. M., et al. (2019). Global spatial risk assessment of sharks under the footprint of fisheries. Nature 572, 461-466.

R Development Core Team (2018). R: A Language and Environment for Statistical Computing. Vienna: R Foundation for Statistical Computing.

Rakison, D. H. (2018). Do 5-month-old infants possess an evolved detection mechanism for snakes, sharks, and rodoents? J. Cogn. Dev. 19, 456-476. doi: 10.1080/15248372.2018.1488717

Roff, G., Brown, C. J., Priest, M. A., and Mumby, P. J. (2018). Decline of coastal apex shark populations over the past half century. Commun. Biol. 1:223.

Schultz, P. W., and Zelezny, L. (2003). Reframing environmental messages to be congruent with American values. Hum. Ecol. Rev. 10, 126-136.

Scruggs, L., and Benegal, S. (2012). Declining public concern about climate change: can we blame the great recession? Glob. Environ. Chang. 22, 505-515. doi: 10.1016/j.gloenvcha.2012.01.002

Sebatier, E., and Huveneers, C. (2018). Changes in media portrayal of humanwildlife conflict during successive fatal shark bites. Conserv. Soc. 16, 338-350.

Simpfendorfer, C. A., Heupel, M. R., White, W. T., and Dulvy, N. K. (2011). The importance of research and public opinion to conservation management of sharks and rays: a synthesis. Mar. Freshw. Res. 62, 518-527.

Skubel, R. A., Shriver-Rice, M., and Maranto, G. M. (2019). Introducing relational values as a tool for shark conservation, science, and management. Front. Mar. Sci. 6:53. doi: 10.3389/fmars.2019.00053

Steel, B. S., Smith, C., Opsommer, L., Curiel, S., and Warner-Steel, R. (2005). Public ocean literacy in the United States. Ocean Coast. Manag. 48, 97-114. doi: 10.1016/j.ocecoaman.2005.01.002

Stevens, J. D., Bonfil, R., Dulvy, N., and Walker, P. (2000). The effects of fishing on sharks, rays, and chimaeras (chondrichthyans), and the implications for marine ecosystems. ICES J. Mar. Sci. 57, 476-494. doi: 10.1006/jmsc.2000.0724

Tavares, D. C., Moura, J. F., Acevedo-Trejos, E., and Merico, A. (2019). Traits shared by marine megafauna and their relationships with ecosystem functions and services. Front. Mar. Sci. 6:262. doi: 10.3389/fmars.2019.00262

Thompson, T. L., and Mintzes, J. J. (2002). Cognitive structure and the affective domain: on knowing and feeling in biology. Int. J. Sci. Educ. 24, 645-660. doi: 10.1080/09500690110110115

Treves, A., and Karanth, K. U. (2003). Human-carnivore conflict and perspectives on carnivore management worldwide. Conserv. Biol. 17, 1491-1499. doi: 10. 1111/j.1523-1739.2003.00059.x

Tsoi, K. H., Chan, S. Y., Lee, Y. C., Ip, B. H. Y., and Cheang, C. C. (2016). Shark conservation: an educational approach based on children's knowledge and perceptions toward sharks. PLoS One 11:e0163406. doi: 10.1371/journal. pone.0163406

United Nations (2018). Revised Roadmap for the UN Decade of Ocean Science for Sustainable Development, Version 2.0. Paris: UNESCO, 51.

Venables, W. N., and Ripley, B. D. (2002). Modern Applied Statistics with S, 4th Edn. New York: Springer.

Veríssimo, D., Challender, D. W. S., and Nijman, V. (2012). Wildlife trade in Asia: start with the consumer. Asian J. Conserv. Biol. 2, 49e50. 
WCPFC (2018). Summary Report of the Technical and Compliance Committee of the Commission for the Conservation and Management of Highly Migratory Fish Stocks in the Western and Central Pacific Ocean, $14^{\text {th }}$ Regular Session, Majuro, Republic of Marshall Islands, September 24-October 2. Available online at https://www.wcpfc.int/system/files/TCC14\%20Summary\% 20Report\%20Final_30\%20Nov.pdf (accessed November 11, 2019).

Whatmough, S., Putten, I. V., and Chin, A. (2011). From hunters to nature observers: a record of 53 years of diver attitudes towards sharks and rays and marine protected areas. Mar. Freshw. Res. 62, 755-763.

Wiernik, B. M., Ones, D. S., and Dilchert, S. (2013). Age and environmental sustainability: a meta-analysis. J. Manag. Psychol. 28, 826-856. doi: 10.1108/ jmp-07-2013-0221

Wirsing, A. J., Heithaus, M. R., and Dill, L. M. (2007). Fear factor: do dugongs (Dugong dugong) trade food for safety from tiger sharks (Galeocerdo cuvier)? Oecologia 153, 1031-1040. doi: 10.1007/s00442-007-0802-3

Worm, B., Davis, B., Kettemer, L., Ward-Paige, C. A., Chapman, D., Heithaus, M. R., et al. (2013). Global catches, exploitation rates, and rebuilding options for sharks. Mar. Pol. 40, 194-204. doi: 10.1016/j.marpol.2012. 12.034
Wright, A. J., Veríssimo, D., Pilfold, K., Parsons, E. C. M., Ventre, K., Cousins, J., et al. (2015). Competitive outreach in the 21st century: why we need conservation marketing. Ocean Coast. Manag. 115, 41-48. doi: 10.1016/j. ocecoaman.2015.06.029

Yokoi, H., Ijima, H., Ohshimo, S., and Yokawa, K. (2017). Impact of biology knowledge on the conservation and management of large pelagic sharks. Sci. Rep. 7:10619.

Conflict of Interest: The authors declare that the research was conducted in the absence of any commercial or financial relationships that could be construed as a potential conflict of interest.

Copyright (C) 2020 Afonso, Roque, Fidelis, Veras, Conde, Maranhão, Leandro and Hazin. This is an open-access article distributed under the terms of the Creative Commons Attribution License (CC BY). The use, distribution or reproduction in other forums is permitted, provided the original author(s) and the copyright owner(s) are credited and that the original publication in this journal is cited, in accordance with accepted academic practice. No use, distribution or reproduction is permitted which does not comply with these terms. 